\title{
A self-guided journey to health
}

\author{
Navigating Canada's health care: $A$ user \\ guide to getting the care you need \\ Michael Decter and Francesca Grosso \\ Penguin Group; 2006 \\ 358 pp $\$ 26$.oo ISBN 0-14-305045-2
}

$\mathrm{T}$ hrough a life cycle approach, this book coaches readers to self-advocate as they navigate through Canada's health care system. Advice, as well as how to find it, is blended into chapters which are organized for pregnancy and birth, childhood, midlife, chronic disease in later life and end-of-life care. The authors encourage the reader to open up their laptop and download the best software available, thus taking over a role usually performed by a family doctor. As we have too few family doctors across Canada, the book notes that Canadians had better equip themselves to be their own advocate.

It is interesting to see the recurring themes of the importance of a family doctor and the importance of a well maintained health record. The purported lack of both of these throughout each life stage is discussed. Readers are urged to get paper, pencils, questions and, better still, a laptop ready when they do go to the doctor. Once there, they are instructed to record every transaction and copy every consultant letter, as our system does not have the ability (or will) to communicate within itself. As a primary care provider, I feel like this is my job description, not my n patients'. However, being a pragmatist, I agree that the book's advice may be a good idea for most Canadians.

Advice given in the book is accurate and appropriate for most patients, although it is perhaps attempting to do more than is possible in its 350 pages.

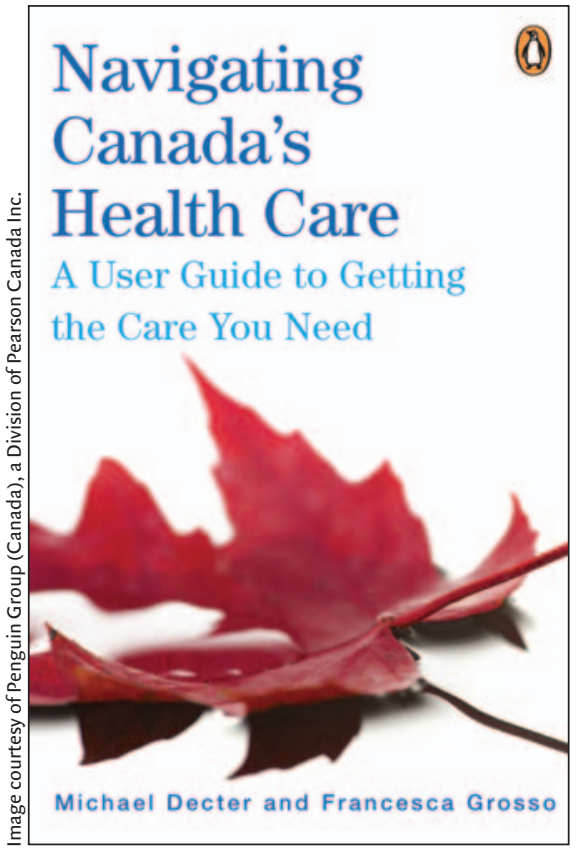

It advises the reader on how to choose a pediatrician or family doctor, acknowledging that this alone is not possible for most Canadians. It offers specific advice on a few common health related topics at each life stage safety issues for children, mental health issues among adolescents, healthy eating, fitness, smoking cessation to name but a few. Key resources are listed at the end of each chapter. However, sometimes they are not relevant to Canada and sometimes a specific topic may not be included. As each chapter could easily be a book in its own right, the advice is more of an overview than an in-depth study.

The authors spend a good deal of time describing how to navigate health insurance schemes, supplemental insurance and how to understand the tax breaks of health care expenses that are not insured. This again is a very practical and pragmatic overview. The
pan-Canadian discrepancies in available support are eye opening. After reading of the inequities across provinces, I thought it might be wise for patients to decide to move based on their individual health service requirements. This book will help readers choose their destination.

"Information is power" and self advocacy are key messages. Thankfully, the authors also stress the importance of considering the appropriateness of the tests that are being self-advocated.

Again, I couldn't help thinking that advocacy on behalf of our patients is the family doctor's role. The problem we come back to is that there aren't enough family doctors. I already appreciate the advantage that a good electronic medical record has to offer, and I would love to work within a health care system that is transparent, easy to navigate, includes excellent inter-professional communications, easy test bookings and well-informed patients who know the system and how to navigate it. A system where no wait time strategies would be needed and my role as patient advocate would be maximized. Valuable patient-care time would not be wasted begging for favours to get sick people booked for tests and consultations that they need (or believe they need after reading this book). This would free my time to be the patient advocate I was trained to be. This book helps patients to selfadvocate at a time when it is difficult to find a family doctor. It does not replace the need for more family doctors.

\section{Janet Dollin}

Family physician

Associate professor

University of Ottawa

Ottawa, Ont. 